\title{
Software Agent Structure for Performance Index Improvement of Cellular Network
}

\author{
Megha Kamble, Roopam Gupta \\ Department of Information Technology, University Institute of Technology, R.G.P.V., Bhopal, India \\ Email: meghakamble@gmail.com
}

Received 14 June 2014; revised 14 July 2014; accepted 30 July 2014

Copyright (C) 2014 by authors and Scientific Research Publishing Inc.

This work is licensed under the Creative Commons Attribution International License (CC BY). http://creativecommons.org/licenses/by/4.0/

\begin{abstract}
Efficient reuse of limited radio spectrum is vital issue to support increasing number of mobile terminals and heterogeneous traffic scenarios. Dynamic channel allocation (DCA) technique is suitable to solve the problem. The drawback of dynamic channel allocation is it may upgrade performance of one cluster and degrade performance of other cluster in large scale cellular network. To balance performance of clusters and increase carried traffic in network, there is need of enhancement of DCA techniques. To introduce improvement in the dynamic channel approach, the paper suggested Multi Agent System (MAS) of physical agents ported at base stations working on the principle of cooperative negotiation to improve the QoS of the network. We formulated an integrated framework which includes fundamental mechanism of call admission control and resource management using hybrid channel allocation (HCA). To balance performance index of various clusters of network, agent negotiation is executed. Our simulation results show that it is possible to significantly enhance performance index of network due to MAS-HCA approach when compared with ES based and ILP based HCA schemes proposed in literature.
\end{abstract}

\section{Keywords}

Cellular Network, Hybrid Channel Allocation, Multi Agent System, Agent Negotiation

\section{Introduction}

A cellular system is considered as network of mobile users (also called as hosts), in the geographical area divided into small hexagonal shapes called cells. The hosts are moving from one cell to another cell and support voice communication. The communication within every cell is controlled by fixed base station (BS), and BSs are connected to central switching office MSC [1]. The communication is supported by frequency channels which are the only resources for all the services. Advanced generations of cellular networks and hosts are also 
supporting variety of services like data and multimedia. This is increasing usage of the network where as resources are scarce. Resource management is needed to cope with the limited frequency spectrum and different traffic densities and scenarios. So still channel allocation is the key problem in cellular network. Due to non availability of channel new call is blocked and, handoff call is dropped. This affects QoS of cellular network.

Call admission control is entry level mechanism of resource management. This is the process of admitting the call only when resource allocation is guaranteed. There are different channel allocation strategies like Fixed Channel Allocation (FCA), Dynamic Channel Allocation (DCA) and Hybrid Channel allocation (HCA). FCA assigns predefined static channel set to every cell where as DCA assigns shared resources on dynamic demand basis. In HCA complete channel set is divided into two sets, static and dynamic in optimal ratios. Previous work of cellular system has presented several variations of these channel allocation schemes [2] and also published results about implementation and improvement of the performance in terms of QoS parameters-call blocking probability, call dropping probability, failure rate, degree of coldness [2]. Recent work in literature has also proposed multi agent system approach for networks [3] so as to provide more autonomy and flexibility to the base stations [4]. However, most of the work of multi agent system is based on distributed dynamic channel allocation. Limitation of purely distributed approach is individual base station gets benefited but overall performance is overlooked. Limitation of DCA is greedy cell grabs more resources. So sometimes this gives rise to hot and cold spots due to variation of degree of coldness (ratio of available channels to allocated channels) from threshold (optimum static value based on ratio of channels). Then to achieve average degree of coldness above threshold for stability of network, negotiation of resources is done by explicit exchange of messages by base station.

To address these issues of channel allocation, Discrete Event Simulation and Multi agent system are possible simulation technologies. Multi agent system is found suitable after analysis, to handle real-time and concurrent events in cellular network like call arrival, call termination, call reallocation in different cells, user mobility. This paper introduces an integrated framework developed for cellular network comprising call admission control and resource management-key components of network performance. The framework is in the form of multiagent system. The important features of the proposed framework are described as follows:

- Layered architecture of agent to handle events in concurrent manner using spatial parallelism.

- Fuzzy logic based entry level control mechanism which reduces call blocking and dropping probability and increases carried traffic.

- Modified Hybrid Channel allocation where agents negotiate for the shared resources at central pool.

- Agent negotiation to support hot cluster having critical dynamic bandwidth requirement.

The paper suggests call admission control (CAC) based on fuzzy logic in first layer. Fuzzy CAC reduces complex mathematical computation and handles uncertainty of parameters needful for CAC. Resource management proposed in this paper is using hybrid channel allocation algorithm. The algorithm is executed in two phases first phase is cooperative agents sharing common resource pool and second is agent negotiation to transfer channel from less loaded cell to heavily loaded cell.

The achievement is improvement in QoS parameters call blocking probability (probability of rejection of calls due to non availability of channel) and call dropping probability (probability of call dropped out of total calls admitted) while maintaining proper resource utilization throughout the network. Also increment in carried traffic and average degree of coldness above threshold is observed for cluster.

The paper is organized as follows. Section 2 provides review of existing literature and its limitations. Section 3 describes and elaborates proposed model. Section 4 presents simulation results followed by conclusion.

\section{Review}

Papazoglou P. M., Karras D. A., Papademetriou R. C. [1] [2] have presented, a novel modelling methodology for supporting wireless network services based on MAS technology and instead of involving physical entities as agents in the MAS system, the authors proposed, to model concurrent network procedures (services), which are more abstract entities, as agents in the proposed simulation methodology.

The literature provides two separate approaches for resource management and call admission control using multi agent system. The literature also provided soft computing approaches for wireless networks [3] [4]. Reference [5] suggested Integer linear programming approach for hybrid channel allocation and presented better results as compared to previous hybrid channel allocation approach for call blocking probability. 
Jamal Elhachmi, Zouhair Guenoun [6] have presented the approach based on self-organizing MAS to solve distributed dynamic channel allocation where all constraints like co-channel, co-site are known to agents. They tested the approach on PHEADEPHIA benchmarks of frequency assignment problem.

One of the early stage and comprehensive simulation model for wireless cellular networks has been built by E. L. Bodanese [7] in order to propose a distributed channel allocation scheme using intelligent software agents. In this study, intelligent collaborative software agents give autonomy to BSs, and modeled several aspects of infrastructure and operations to increase the network robustness and improve resource allocation.

A MAS for resource management in wireless mobile multimedia networks is presented in [8]. According to [8], the final decision for call admission is based on the participation of neighbour cells. Thus an agent runs in each cell or Base Station (BS). The authors have presented that the call dropping probability can be reduced while average bandwidth utilization can be increased with multi agent system. The work in [9] shows cooperative negotiation in a MAS for supporting real-time load balancing of a mobile cellular network. In the above proposed MAS, agents are modeled as different service providers in the market and as network operators that manage the radio resource of different network regions. According to [9], agent communication is achieved through messages and negotiations are fruitful for decision making. Agents are negotiating for adjusting dynamically the cell shape and size and for balancing the traffic load over the network in [9].

Proposed framework integrates the call admission control and channel allocation strategy using layered architecture, to further enhance the output. Proposed model also utilizes property of agents negotiation for sharing common resources. The literature provides DCA and FCA as resource management schemes with MAS. Drawback of this approach is demand based resource allocation and demands are handled using ordering of the timestamp. So if we consider a simple scenario, where demand request of low traffic cell is having low timestamp as compared to heavy traffic cell, it will be fulfilled causing call block/drop in hot cell. Second limitation is channel borrowing is preserving only individual benefit, overall performance is overlooked. The proposed framework tries to provide mechanism of resource allocation on need basis. It works on the principle that individual cell's performance should be upgraded and cold or lender cell and it's cluster should not be affected. To handle concurrent events, multi agent system based framework is preferred.

\section{Proposed Methodology}

Proposed framework integrates a fundamental mechanism of call admission control and resource managementkey components used for quality of service (QoS) provisioning in a network. Call admission control works for allowing the calls upto the extent such that QoS parameters will not be affected in individual cell as well as that of cluster. The diagrammatic representation of the proposed work is shown in the Figure 1. The layered architecture adapted for Multi Agent system and functional architecture is elaborated in [10] [11]. The following section discusses about the layers.

\subsection{Reactive Layer (RL)}

When a call is generated or entered in cell, reactive layer of base station agent determines whether to accept or reject the call using fuzzy logic based mechanism. The proposed fuzzy CAC consists of three phases:

- Fuzzification: the parameters network load, traffic load, dynamic channel requirement and user precedence contributes to admission decision. These parameters are represented by membership function using different fuzzy sets.

- Fuzzy inference engine: This phase consists of development of fuzzy rules where there will be combination of all the four parameters to determine admission decision. It is defined in linguistic terms Strongly Accept, Strongly reject, weakly accept and buffer, weakly reject and buffer.

- Defuzzification: This phase converts output from linguistic term into crisp output using mean of maxima method. More details of fuzzy logic implementation at reactive layer are presented in [10] and experimental results are included in Section 3 in this paper. Novelty of the work lies in redesigning the fuzzy logic parameters [11] and using defuzzification formulae for call buffering delay parameter.

\subsection{Local Planning Layer (LPL)}

Local planning layer is working with the help of hybrid channel allocation which is combination of Fixed Channel allocation and Dynamic Channel Allocation scheme [12]. Benchmark set of 70 channels are used for 


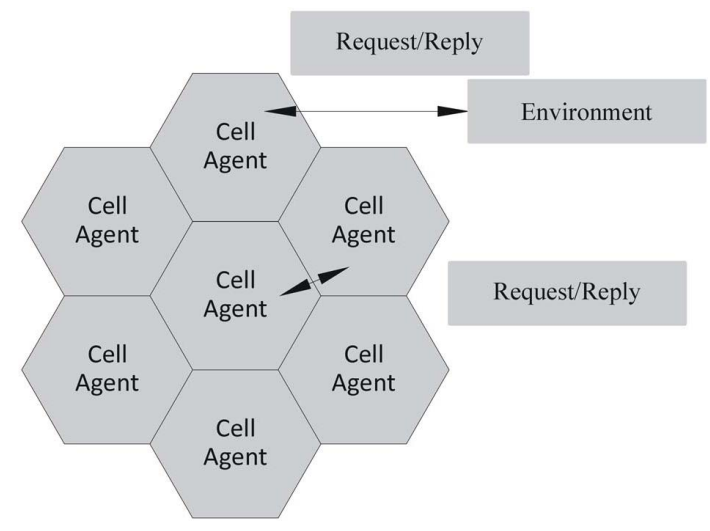

Figure 1. MAS for cellular network.

simulation purpose in three representative optimal ratios vise 21:49, 49:21, 35:35 (the ratio for fixed and dynamic channels FC:DC) [13]. Reactive layer tries to allocate a channel from statically allocated channel set. If no channel is available in static set, call request is transferred to local planning layer, for channel borrowing. Similarly buffered calls in reactive layer are processed by hybrid channel allocation in local planning layer.

Proposed work assumes hexagonal cellular network model shown in the Figure 3, representing large scale network with 60 degree omni directional antenna pattern. For analysis purpose, it is divided into clusters as shown in the Figure 2. Cluster is compact pattern of a cell at center, surrounded by adjacent cells. Number of cells in cluster is related to minimum reuse distance [7].

General representation of cellular network model [14] is an array of $n$ rows and m columns as shown in Figure 3. Every cell is represented as $(i, j)$ indicating its position at row $i$ and column $j$.

By Euclidian Distance formula, minimum reuse distance between any two cells $(0,0)$ - $(i, j)$ is calculated by equation

$$
D=\sqrt{i^{2}+i \cdot j+j^{2}}
$$

Number of cells in cluster $[2]=\mathrm{N}=\mathrm{D}^{2}$

Nearest co channel cells can be found for any cell, by turning along any hexagonal chain in the array, by 60 degree and moving $i$ cells in the direction and then moving $j$ cells along the direction by turning again in 60 degree, so $i$ and $j$ are called as shift parameters. Mercedes Logo structure is adopted from [7] to calculate number of cells in cluster where values of shift parameters are $i=2, j=1$. With these values, number of cells in cluster is seven and number of nearest cochannel cells are six in clockwise or anticlockwise direction.

To study proposed HCA, static set of channels are assigned to each cell where channels are numbered $i, i+$ $2 \mathrm{~N}, i+3 \mathrm{~N}$ and the channels are separated so as to minimize adjacent channel interference [2] with the help of omnidirectional antenna pattern. Sample set of clusters with the cells containing traffic rate, cell number and static channel set for 2149 ratio are shown in the Figure 2.

\subsection{Proposed Hybrid Channel Allocation}

In HCA, the total set of available channels for the network is subdivided into two sets-fixed channel set (FC) and dynamic channel set (DC). Proposed simulation is using total 70 channels, divided into ratios 21:49, 49:21, 35:35 (FC:DC) proposed in [13] [15] [16].

- For any FC:DC ratio, initially $\mathrm{FC}_{\mathrm{i}}$, a subset of FC represents set of static channels allocated to individual cell $\mathrm{i}$, is containing $F C / N$ where $\mathrm{N}$ is cluster size and $\mathrm{N}=7$ in proposed work.

- $\mathrm{BUSY}_{\mathrm{i}}$, a subset of $\mathrm{FC}_{\mathrm{i}}$ represents the set of channel assigned to a call to support communication session at a particular time. When a new or handoff call originates in Cell $\mathrm{C}_{\mathrm{i}}$, one of the available channels in $\mathrm{FC}_{\mathrm{i}}$ is allocated to call, it is transferred from $\mathrm{FC}_{\mathrm{i}}$ to $\mathrm{BUSY}_{\mathrm{i}}$.

$$
\mathrm{FC}_{\mathrm{i}}=\mathrm{FC}_{\mathrm{i}}-1, \mathrm{BUSY}_{\mathrm{i}}=\mathrm{BUSY}_{\mathrm{i}}+1, \mathrm{BUSY}_{\mathrm{i}} \in \mathrm{FC}_{\mathrm{i}}
$$

- If no channel is available in $\mathrm{FC}_{\mathrm{i}}$, it is checked that whether there is possibility to get a free static channel af- 


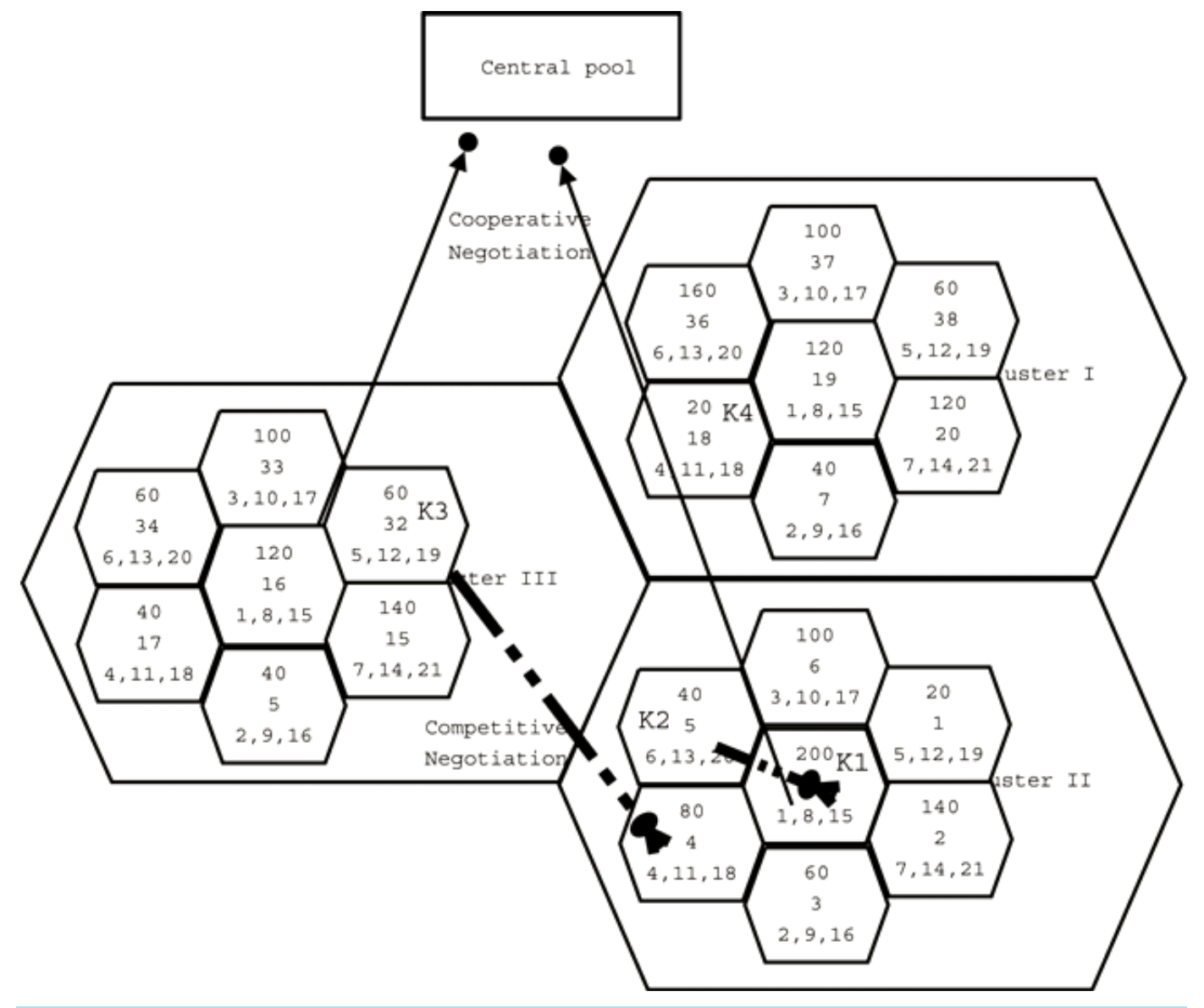

Figure 2. Cooperative negotiations of agents.

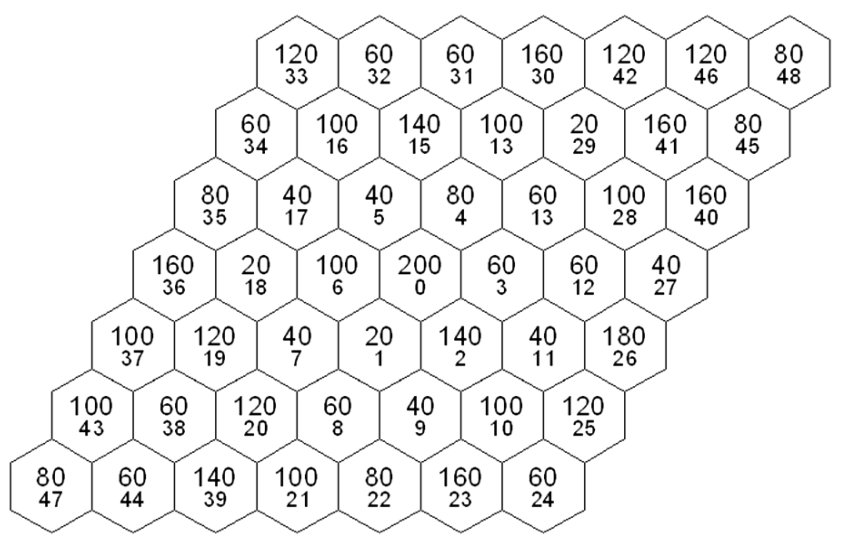

Figure 3. Non uniform traffic pattern.

ter some delay (predefined delay less than maximum interhandoff delay i.e. $10 \mathrm{msec}$ ), otherwise request is sent to DC for resource negotiations from shared pool. DC checks suitable interference free channel and allot it to requesting cell.

- The channel is temporarily allocated to the cell to support the session and after completion of session returned back to DC. Base station can retain the channel in case it's cluster is becoming hot spot during the time interval when channel is supporting communication session.

- If there is no suitable channel available at DC, in case of new request, call is blocked and if request is handoff request, it is dropped. 
Our proposed work is simulated using OmNet discrete event simulator. Simulation model is based on some parameters and following variables are used to handle parameters.

- $\mathrm{T}_{\mathrm{i}}$ is the clock value maintained at Cell $\mathrm{C}_{\mathrm{i}}$ to record each event. Simulation starts with smallest interarrival time amongst all the cells. So initially $\mathrm{T}_{\mathrm{i}}$ is zero or smallest interarrival time (unit of time in simulator equal to msec).

- $\mathrm{T}_{\mathrm{i}}=$ simTime() function of simulator returns current time and is stored in $\mathrm{T}_{\mathrm{i}}$ when any event like call arrival, call departure, call termination, channel request, request reject occurs.

- Several call requests are originated in cell concurrently, they are stored by variable msg. msg consists of several attributes.

○ $m s g \rightarrow$ timestamp: timestamp of call arrival

$\circ \mathrm{msg} \rightarrow$ priority: priority of call either new(0) or handoff(1)

$\circ \mathrm{msg} \rightarrow$ channelno: sequence number of channel allocated from DC

- serviceTime: mean service time of a call. Default value is $180 \mathrm{sec}$.

- txTime: time required to fetch a channel from central pool. Default value is $2 \mathrm{msec}$.

To implement proposed algorithm, following counters are declared:

- Arrived $_{\mathrm{i}}$ : to store number of calls arrived

- Processed $_{\mathrm{i}}$ : to store number of calls processed after allocation of channel

- Blocked $_{\mathrm{i}}$ : to store number of calls blocked due to non availability of channel initially

- Dropped $_{\mathrm{i}}$ : to store number of calls dropped when call was in progress in the region

- Queued ${ }_{\mathrm{i}}$ : to store number of calls queued while waiting for channel to be allocated

- Expdept: a queue to store expected timestamp when allotted fixed channel will be freed.

The following steps are executed in the algorithm:

- Once Fuzzy CAC in Reactive Layer accepts a call, it is to be set up in Cell i, the following actions are taken by BSi agent's local planning layer:

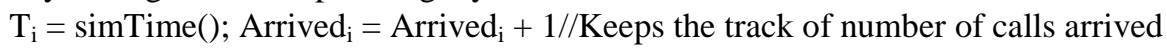

if $\left(\mathrm{FC}_{\mathrm{i}} !=\varnothing\right.$ and threshold $\left.\leq\left|\mathrm{FC}_{\mathrm{i}}\right|\right)$

begin

Busy $_{\mathrm{i}}=$ Busy $_{\mathrm{i}} \cup\{\mathrm{f}\}$ where $\mathrm{f} \in \mathrm{FC}_{\mathrm{i}}$;

Processed $_{\mathrm{i}}=$ Processed $_{\mathrm{i}}+1$;

end

else

Send timestamped REQUEST message to Central Pool

end

Expected departure timestamp of the call is calculated using $T_{i}$ and serviceTime and inserted into queue Expdept $\mathrm{t}_{\mathrm{i}}$.

$/ *$ threshold is minimum number of channels reserved for handoff calls. We assume threshold $=1$ for FC:DC ratio 21:49 and 35:35. We assume threshold $=2$ for FC:DC ratio 49:21 [16].*/

- Simulator Parameter txTime stores time required to fetch a channel from central pool DC. When there is no availability of channel in $\mathrm{FC}_{\mathrm{i}}$ and request is to be sent to DC, base station agent pops element in queue Expdept $_{\mathrm{i}}$ in FIFO order.

- Decision Parameter dp is calculated as dp $=\max \left(\right.$ txTime, $\left._{\text {Expdept }} \rightarrow \operatorname{pop}()\right)$. This decision parameter determining factor to decide whether static channel will be reused or dynamic channel will be transferred from DC. It should be less than maximum tolerable interhandoff delay $10 \mathrm{~ms}$ [16].

- Decision parameter is compared with channel acquisition delay taken by agent (also referred as agent decision delay variable) which will be specified to simulator and can be static or dynamic. We performed experiments for number of call arrival rates and number of values for decision parameter values in the range of 0.5 to $10 \mathrm{msec}$. Experiments are carried out for one hour duration by assigning static and dynamic values to agent decision delay ( $a d d v$ ) variable.

$\circ$ if $\mathrm{dp}>a d d v$, request is sent to DC for channel otherwise queued

○ If DC is Ø or $|\mathrm{DC}|<$ threshold, blocked $_{\mathrm{i}}=$ blocked $_{\mathrm{i}}+1$

$\circ$ if $d p<a d d v$, queue the call, queued $_{\mathrm{i}}=$ queued $_{\mathrm{i}}+1$

$\circ$ if the call arrived is handoff, all the above steps are repeated and if $\mathrm{dp}>a d d v, \operatorname{dropped}_{\mathrm{i}}=\operatorname{dropped}_{\mathrm{i}}+1$

$\circ$ if (simTime ()$>($ Expdept_i $\rightarrow$ pop()) it indicates fixed channel is freed, the freed channel is allotted to 
queued call, step ii is repeated and counter is updated with queued ${ }_{i}=$ queued $_{i}-1$

DC receives the request and compares the consecutive requests using msg parameters timestamp, priority and last channel allocated to base station.

In traditional HCA, channel from DC is transferred as per demand (ordered timestamp) and availability of channels in DC. The limitation of the mechanism is, DC cannot support the request from BS due to non-availability of suitable interference free channel. When there are multiple requests, cell in need can not be given priority as traditional HCA is prioritised as per demand.

In Modified HCA, DC stores assigned channel to base station in separate array. So for new request from BS, on non availability of suitable channel from available channels at DC, it is checked in BS array at DC whether there is possibility that same busy or alloted channel to BS will be freed after a short while and that will be more suitable channel for requesting BS.

The BS agent with critical dynamic requirement and due to high call arrival rate cannot afford waiting, is termed as needy agent over the BS agent with moderate call arrival rate, can afford waiting for resource. So less needy BS agent shows cooperative negotiation and needy BS agent gets benefited. Due to use of multi agent system, it is possible that agents negotiate with cooperation for the resources from common pool as per needy situation instead of demand. Once alloted channel to BS is reutilised due to cooperative agents in turn increasing resource utilization.

DC transfers the channel to request, in such a manner that following hard constraints and soft constraints [13] are not violated.

- Cochannel Constraint: Same channel can be assigned to two cells separated by minimum reuse distance.

- Adjacent channel constraint: Channels in the neighboring cells must be separated by minimum amount.

- Cosite Channel Constraint: Channels assigned in a cell must be separated by minimum amount. $\operatorname{diff}_{\mathrm{d} 1, \mathrm{~d} 2}>\mathrm{N}$ where $\mathrm{d} 1$ and $\mathrm{d} 2$ are resources in a single cell

Proposed model also follows soft constraints [13]

- Packing condition: use minimum number of channels by waiting for the channel already in use. On nonavailability of direct suitable channel, call is delayed and already alloted channel to cell is reused.

- Resonance Condition: assign same channel to the cell that belong to same reuse scheme. All the Channels in DC are numbered and while allotting channel their sequence number is compared with that in Busy $y_{i}$ set of Cell . $_{\text {. }}$

Thus far, most work of resource allocation using FCA, DCA and HCA has focused on resource allocation if available to the cell without much concern about maximum reuse of individual channel and cell's status and channel's occupancy. To improve this allocation, we propose a model where utility of an agent can be derived on the basis of benefit agent receives by using shared resource from set of resources, but the benefit is reduced by the effect of call delaying for time equivalent to agent level decision delay which is short delay.

\subsection{Network Agent's Utility}

Our model is applied for resource allocation with sharable and non-sharable resources [17]. This problem is defined with a triplet $(C, R, U)$, where $C$ is set of finite agents representing cells, $R$ is set of finite resources. $U$ is resource utilization. Fourth component in this problem is defined by agent's utility, for allocating resource from set $R$ for each agent. A solution to allocation problem is the resource allocation that satisfies the parameters like channel utilization and reusability of individual resource, failure rate and average degree of coldness of cluster near threshold. Fourth component in this problem is defined by agent's utility, for allocating resource from set $R$ for each agent and which defines the solution.

The utility of an agent is expressed as:

$$
\mathrm{v}_{\mathrm{i}}(\mathrm{t})=\mathrm{u}_{\mathrm{i}}(\mathrm{t})-\text { delay }_{\{\mathrm{i}, \mathrm{r}\}}
$$

where Delay in allocating resource $r$ from DC to agent $i$ is expressed as delay fi,r\} $_{\text {. }}$.

Let $\mathrm{R}(\mathrm{t})$ denotes the total amount of the resource that required at time $\mathrm{t}$ in a cellular system so as to satisfy the requests. Note that $\mathrm{R}(\mathrm{t})$ changes dynamically with the time $\mathrm{t}$. Among $\mathrm{R}(\mathrm{t})$, let us assume that $\mathrm{X}_{\mathrm{i}}(\mathrm{t})$ amount of the resource are required at cell $\mathrm{i}$ and $\mathrm{N}$ is the total resources available in cellular system.

Let $R_{i}(t)$ denote resources available at time $t$ in cell $i$.

Let $\mathrm{u}_{\mathrm{i}}(\mathrm{t})$ denote the utilization of the resource at cell $\mathrm{i}$ at time $\mathrm{t}$. 


$$
\mathrm{u}_{\mathrm{i}}(\mathrm{t})=\mathrm{X}_{\mathrm{i}}(\mathrm{t}) / \mathrm{R}_{\mathrm{i}}(\mathrm{t})
$$

The value greater than 1 indicates call blocking or dropping. That is, agent i improves it's performance by allocation of resource from $R$, but this gain is reduced by short delay is allocation. Value of agent's utility less than 1 indicates reduction in failure rate.

\subsection{Cooperative Layer (CPL) Negotiation}

The following section describes multi agent interaction for resource allocation for the needy cell which plays the most important role in cellular network performance. Network congestion is observed over a cell for 60 sec [1]. If degree of coldness of cell is less than threshold over this period, the cell is referred as needy cell that triggers cooperative layer negotiation. We assume two scenarios-needy cell can be part of complete hotspot or incomplete hotspot. A cluster in which 3 - 4 cells out of 7 have degree of coldness less than threshold, is treated as hot spot. Categorisation of a cluster as complete or incomplete hotspot is done by observing spot density (number of hot cells in cluster). If all the cells in cluster are hot, it is called as complete hotspot otherwise incomplete. In this scenario, hot cells try to attract more resources from common pool.

Due to hard and soft constraints of channel reuse, although channel is available in common pool may not be used properly. Multi agent negotiation [15] in cooperative layer suggests a solution where resources will be reused among cochannel cells and will reduce message complexity of borrowing every time from common pool. This converts HCA to Distributed HCA. We propose co-ordination technique Contract-Net protocol [2] to improve resource allocation to needy cell. Contract-Net protocol can be applied to specific domain using different kinds of algorithms and functions to solve a problem or different parts of a problem. In proposed multi agent framework, needy BS agent plays the role of initiator and base station agents of cochannel and other cold cells are playing the role of responders. Negotiation in this Contract net protocol is using utility function evaluated with co-channel and other cold cells. In proposed model every borrower cell agent can negotiate with minimum 6 cochannel cell agents in clockwise or anticlockwise direction. Probability is $50 \%$ cold cochannel cells participate in negotiation. Utility function is based on following parameters

- degree of coldness of cluster of donor

- degree of coldness of donor cell

- minimum number of resources of common pool DC to support emergency peak traffic hours in network (called as threshold of DC)

These parameters are considered because in this distributed approach, donor cell's interests and performance of it's cluster is taken care by scheme. As well as it is seen that central pool should be there to cater need of other cells.

Refer to Figure 2, Steps followed in traditional HCA

Step 1: New call arrives in Cell K1, (200 calls/hr) but a free channel does not exist and so request is sent to central pool. Channel borrowed from central pool and call is accepted.

Step 2: Handoff call arrives in Cell K3 (60 calls/hr) but a free channel does not exist and so request is sent to central pool.

Step 3: New call arrives in Cell K4 (20 calls/hr) and no free channel exists, channel allotted from central pool.

Step 4: New call arrives in Cell K1 (200 calls/hr), no channel exists locally and no channel in central pool, call is blocked.

Step 5: Handoff call in Cell K1, no channel at central pool or at locally, call is dropped

Step 6: Call terminated in Cell K4 (20 calls/hr), channel is released and available.

Step 7: Call in cell $\mathrm{K} 2$ calls/hr moved out, channel is released.

Steps followed in MA-HCA

Step 1: Call arrival at Cell K1 (200 calls/hr), but a free channel does not exist and so request is sent to central pool. Channel borrowed from central pool and call is accepted.

Step 2: Call arrival in Cell K3 (60 calls/hr) but a free channel does not exist and so request is sent to central pool. Channel is allotted.

Step 3: New call arrives in Cell K4 (20 calls/hr) and no free channel exists, request sent to central pool. Channel is not allotted, but request is queued, as agent decision delay is less than maximum interhandoff delay.

Step 4: New call arrives in Cell K1 (200 calls/hr), channel is allotted from central pool dynamically as the text edit has been completed, the paper is ready for the template. Duplicate the template file by using the Save As 
command, and use the naming convention prescribed by your journal for the name of your paper. In this newly created file, highlight all of the contents and import your prepared text file. You are now ready to style your paper agent decision delay is more than maximum interhandoff delay.

Step 5: Call is terminated in Cell K4 (20 calls/hr), queued call is allotted channel.

Consider clusters Cluster I, Cluster II, Cluster III shown in Figure 2. Cell K1 $\in$ Cluster II is hot cell initiating negotiation. Let k2, k3, k4 are adjacent cold and co channels cells which are participating in negotiation as responder cells and $\mathrm{K} 2 \in$ Cluster II , K3 $\in$ Cluster III, K4 $\in$ Cluster I. Uk $\mathrm{H}_{\mathrm{i}}$ represent utility function of participating responder cells $\mathrm{k} 2, \mathrm{~K} 3, \mathrm{k} 4$. Cell $\mathrm{K}_{1}$ requests utility function from all responders. Some responders participate in negotiation and responds within negotiation tenure equal to maximum interhandoff delay.

The responder that returns maximum value of utility function is the best lender, chosen for borrowing channel to donor. Borrower accepts best proposal and sends request to lender to retain the channel instead of returning it to central pool. Lender transfers the channel to borrower.

$$
\begin{gathered}
\text { Average degree of coldness of cluster }=\sum_{i=1}^{7} \operatorname{deg} \operatorname{cold}(\mathrm{Ci}) / 7 \\
\text { Utility function for responders } \mathrm{U} k i=\frac{\text { degree of coldness of donor cell }(\operatorname{deg} \operatorname{cold}(k i))}{\sum_{i=1}^{7} \operatorname{deg} \operatorname{cold}(\mathrm{Ci}) / 7 \times \text { threshold of central pool DC }}
\end{gathered}
$$

The negotiation will be successful when there will be at least one donor cell with average degree of coldness above threshold and its cluster is cold or moderate. Probability calculation for the attempts where channel will be lent by suitable cell from cold cluster is done with the help of following formula:

$$
\begin{aligned}
& \text { Probability of balanced cluster } \\
& =\frac{\text { wo } \times \text { minimum degree of coldness of donor cell }}{w 1 \times \text { minimum avg degcold }(\text { cluster }) \times \text { threshold of DC }}
\end{aligned}
$$

W0: probability of at least one cochannel or other cold cell is with enough resources;

W1: probability that at least one cluster is available with degree of coldness above threshold.

\section{Simulation and Discussion}

The simulated cellular network consists of a 2D structure of $7 \times 7$ hexagonal cells-a wrapped-around layout array of cells, with each cell having six neighbors. Model is representing microcellular network [1] which is like a city model spread over area of $1 \mathrm{~km}^{2}$. This model represents some areas with heavy traffic during peak hours. Simulation is done for nonuniform traffic pattern shown in Figure 3. Every cell contains traffic rate (upper digit) and cell number (lower digit). Simulation is also done for traffic rate in the range $20-1000$ calls/hr for uniform as well as nonuniform scenario. A set of 70 channels is used for simulation. The arrival of calls at any cell is assumed to be a poisson process. Nonuniform traffic scenario assumes 3 times mean arrival rate as compared to uniform traffic scenario. Cell is always in one of the two states. During peak hours, a cell can change from hot state to cold state and cell remains in cold state for about 30 mins. Simulation parameters are listed in Table 1 and Table 2.

JADE 3.1 (Java Agent Development Environment) is an open source framework and is foundation for Intelligent Physical agent compliant Conclusions (Figure 4). JADE is powerful open source tool to study multi agent system and agent negotiation Agents are communicating with each other through Agent Communication Language (ACL) [17] using message exchange performatives listed in Table 3 during negotiation, for contract net protocol. Figure 5 shows JADE model where proposed software agents are implemented and simulated for cooperative negotiation.

\subsection{Performance Analysis}

The Performance analysis for the cellular network is done in terms of increase in carried traffic, failure rate, call blocking probability (Rb), call dropping probability (Rd) and borrowing attempts at various layers.

Performance index of cellular network is failure rate derived by the formula

$$
\text { failure rate }=R b+(1-R d) \cdot R b
$$


Table 1. Common parameters for uniform and non uniform traffic scenario.

\begin{tabular}{cc}
\hline Parameter & Value \\
\hline Cluster Size & 7 \\
Number of Channels & 70 \\
FC:DC Ratio & $21: 49,35: 35,49: 21$ \\
Mean arrival rate in a cell (poisson arrival) & $\lambda$ \\
Mean service time per communication session & $180 \mathrm{sec}$ \\
Mean interhandoff rate & $1 / 80 \mathrm{sec}$ \\
Mean processing time at BS & $2 \mathrm{msec}$ \\
Mean processing time to borrow channel from DC & $2 \mathrm{msec}$ \\
\hline
\end{tabular}

Table 2. More parameters for non uniform traffic scenario.

\begin{tabular}{cc} 
Parameter & Value \\
\hline Mean arrival rate in a hot cell (poisson arrival) & $3 \lambda$ \\
Mean interhandoff rate in hot cell & $1 / 180 \mathrm{sec}$ \\
Threshold for degree of coldness of cluster & 0.4 \\
Threshold for degree of coldness(partially from literature) & 0.3 \\
$21: 49$ & 0.2 \\
$35: 35$ & 0.14 \\
$49: 21$ & \\
Agent decision delay value & $1 \mathrm{msec}$ \\
Static & $1-10 \mathrm{msec}$ \\
Dynamic & $8 \mathrm{msec}$ \\
Negotiation tenure at central pool & $1-10 \mathrm{msec}$ \\
Static & $3 \lambda$ \\
Dynamic & $1 / 180 \mathrm{sec}$ \\
\hline Mean arrival rate in a hot cell (poisson arrival) & \\
Mean interhandoff rate in hot cell &
\end{tabular}

Table 3. JADE performatives for negotiation.

\begin{tabular}{cc}
\hline Performative & Action \\
\hline REQUEST & Initiator requests for utility function to participate in negotiation \\
PROPOSE & Responders ropose utility function \\
ACCEPT_PROPOSAL & Contract net protocol finds best proposal and message sent to best lender \\
REJECT_PROPOSAL & Message sent to other lenders for rejecting proposal \\
INFORM & Channel is sent from lender to borrower \\
REFUSE & Agents refuse to participate in negotiation \\
\hline
\end{tabular}

Table 4 displays the results for increase in carried traffic due to application of fuzzy CAC at reactive layer. This layer works for fast response of arriving calls. Fuzzy CAC decision making in this layer is useful to determine carried traffic. The calls accepted with linguistic variables strongly accept are processed and the calls with linguistic variable strongly reject are dropped. Admission decision weakly accepts and buffer as well as weakly reject and buffer, determines the calls to be buffered and processed. These calls increase number of calls to be served. Minimum 2.22\% and maximum 16.02\% increase in carried traffic is seen at this layer.

Local planning layer executes modified Hybrid channel allocation to maximize the utilization of static resources. In traditional HCA, number of channels available to network is $|\mathrm{FCi}|+|\mathrm{DC}|$. Proposed MA-HCA provides the number of channels equal to $(|\mathrm{FCi}| \times$ reuse factor $)+|\mathrm{DC}|$. Reuse factor is proportional to ratio of mean 


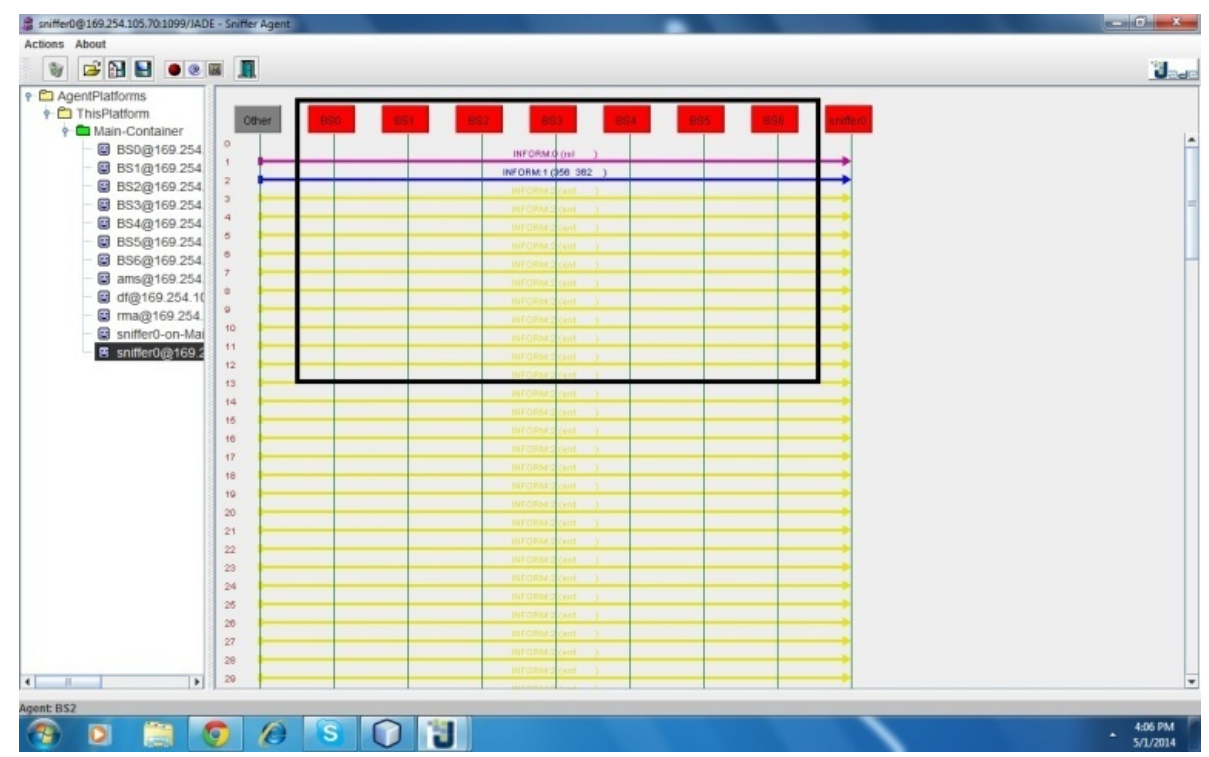

Figure 4. JADE tool simulation model for agent negotiation.

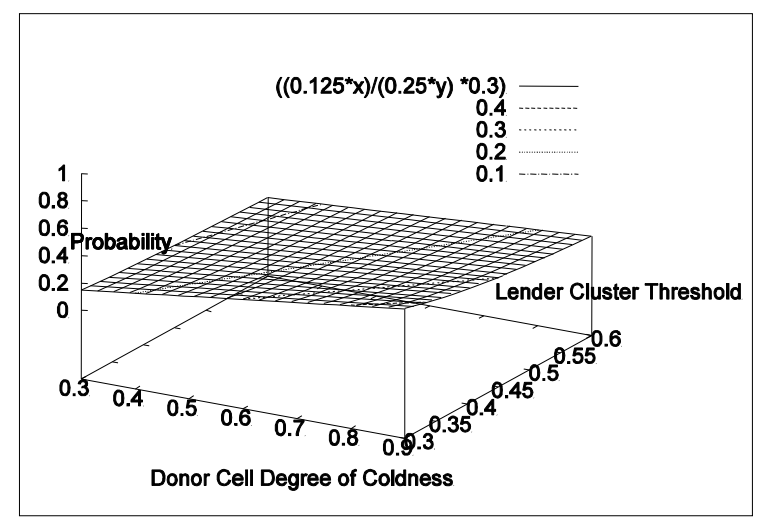

Figure 5. Probability of getting balanced cluster with 2149 in nonuniform scenario.

Table 4. Tabulated result \% increase in carried traffic at RL non uniform traffic scenario.

\begin{tabular}{cccc}
\hline Traffic Profile & 2149 & 3535 & 4921 \\
\hline LOW & 6.47 & 3.06 & 2.22 \\
MODERATE & 16.02 & 4.86 & 5.14 \\
HIGH & 5.93 & 7.29 & 6.74 \\
VERYHIGH & 5.90 & 13.34 & 12.27 \\
\hline
\end{tabular}

service time to mean inter arrival time. MA-HCA avoids static resource to be unutilized. The principle of soft constraint of reusing allotted channel to cell and using minimum channels for the calls in a cell is effectively executed. This increases number of available resources and so serves more number of calls in this layer. Data is presented in the tabular form in Table 5. Minimum increase of $7 \%$ and maximum increase of $100 \%$ is observed in LPL when simulated for all possible traffic profile in case of non uniform traffic.

Number of messages in LPL indicates number of borrowing attempts (BA) from central pool and negotiations (negs) are done at central pool to determine needy agent and priority of resource to needy agent. In nonuniform traffic scenario, with 2149 ratio, more borrowing attempts are done and this is due to less number of static resources available at cell site. Details are shown in Table 6 for uniform traffic and Table 7 for nonuniform traffic scenario. 
Table 5. Tabulated result \% increase in carried traffic at LPL nonuniform traffic scenario.

\begin{tabular}{cccc}
\hline Traffic Profile & 2149 & 3535 & 4921 \\
\hline LOW & 30 & -4 & 100 \\
MODERATE & -16 & 1 & 13 \\
HIGH & 40 & 40 & 7 \\
VERYHIGH & 26 & 50 & 7 \\
\hline
\end{tabular}

Table 6. Tabulated result for borrowing attempts at LPL uniform traffic scenario.

\begin{tabular}{ccccccc}
\hline Traffic Profile & \multicolumn{2}{c}{2149} & \multicolumn{2}{c}{3535} & \multicolumn{2}{c}{4921} \\
\cline { 2 - 7 } & BAs & Negs & BAs & Negs & BAs & Negs \\
\hline LOW & 18 & 16 & 21 & 21 & 20 & 13 \\
MODERATE & 185 & 11 & 201 & 21 & 177 & 26 \\
HIGH & 271 & 42 & 252 & 47 & 241 & 33 \\
VERYHIGH & 343 & 62 & 340 & 63 & 334 & 53 \\
\hline
\end{tabular}

Table 7. Tabulated result for borrowing attempts at LPL nonuniform traffic scenario.

\begin{tabular}{cccccccc}
\hline Traffic Profile & \multicolumn{2}{c}{2149} & \multicolumn{2}{c}{3535} & \multicolumn{2}{c}{4921} \\
\cline { 2 - 7 } & BAs & Negs & BAs & Negs & BAs & Negs \\
\hline LOW & 41 & 25 & 39 & 35 & 32 & 12 \\
MODERATE & 138 & 40 & 191 & 42 & 131 & 43 & 26 \\
HIGH & 295 & 20 & 262 & 44 & 236 & 350 & 26 \\
VERYHIGH & 384 & 75 & 372 & 48 & & & \\
\hline
\end{tabular}

With the help of agent negotiation, the resources are borrowed from neighbors in distributed manner that reduces number of borrowing attempts from central pool. No change is seen in low traffic profile in non uniform traffic scenario where as in rest cases, $5 \%$ to $17 \%$ reduction is seen.

\subsection{Graphical Analysis}

Probability of balanced cluster is highest i.e. $40 \%$ with 2149 ratio, that with 3535 is $30 \%$ and with 4921 is $15 \%$. Agent negotiation for balanced cluster is more effective for 2149 ratio, with $40 \%$ probability utility function will give the proposal to lend the channel (Figure 6 and Figure 7).

Graphical analysis of failure rate for DCA, HCA and MAHCA is shown in the Figure 8 and Figure 9. Average Failure Rate observed with DCA is 0.26 , with HCA is 0.09 and that with MA-HCA is 0.03 for nonuniform traffic scenario. With MA-HCA, Failure rate is decreases by $11 \%$ when compared to DCA and 33\% when compared to HCA. Failure rate with uniform traffic scenario, for DCA is 0.59, HCA is 0.17 and MA-HCA is 0.07. With MA-HCA failure rate is decreased by $11 \%$ when compared to DCA and $41 \%$ reduction is there in comparison of HCA with uniform traffic.

\subsection{Comparison with Existing HCA Approaches}

Finally proposed approach is also compared with existing HCA approaches-evolutionary strategy (ES) and integer linear program (ILP). Results displayed in Table 8 shows that the proposed approach produces better results and shows the significant improvement in call blocking probability parameter.

\section{Conclusion}

Integrated framework for call admission control and resource management is provided. Framework is implemented using multi agent system where agents are cooperating with each other to negotiate resources from common pool in first phase. Second phase negotiation is done to balance degree of coldness of hot as well as 


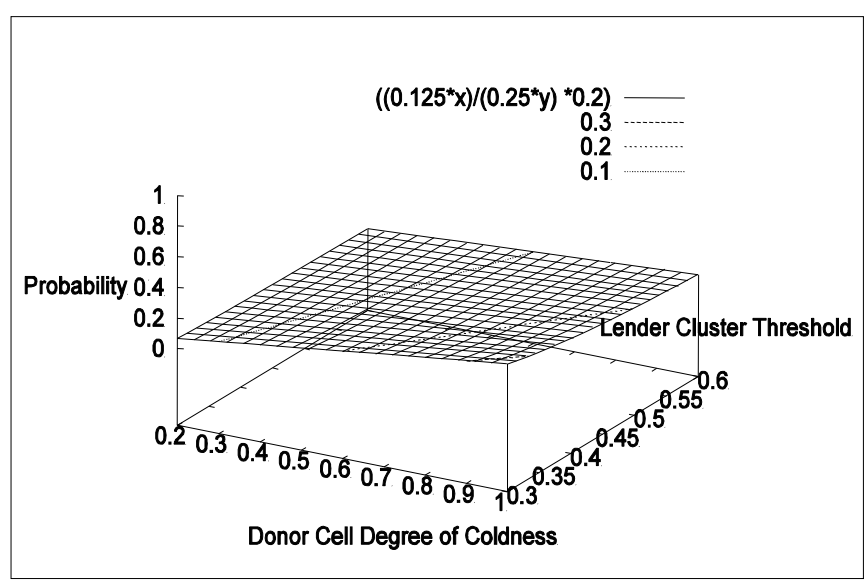

Figure 6. Probability of getting balanced cluster with 3535 in nonuniform scenario.

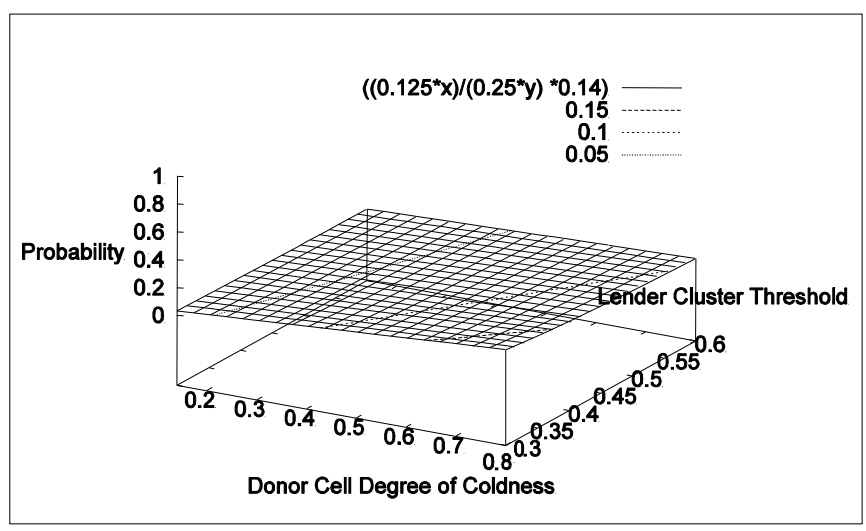

Figure 7. Probability of getting balanced cluster with 4921 in nonuniform scenario.

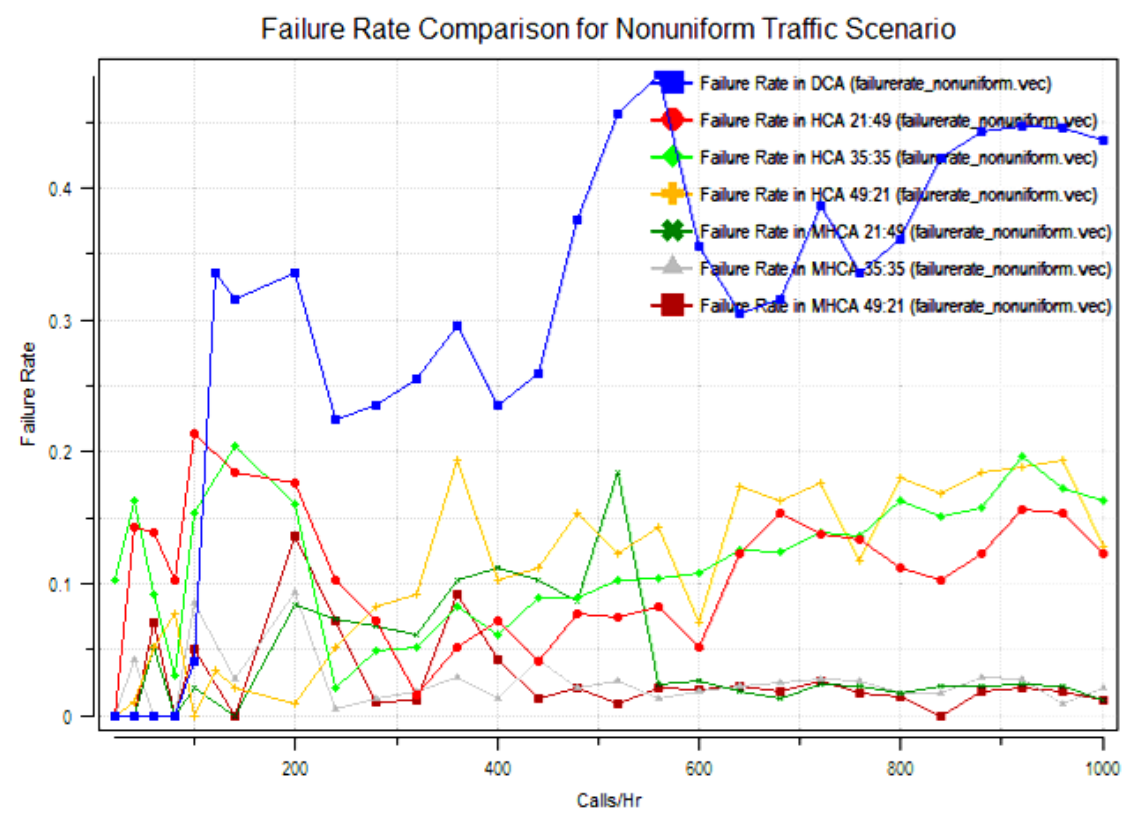

Figure 8. Failure rate comparison for non uniform traffic. 


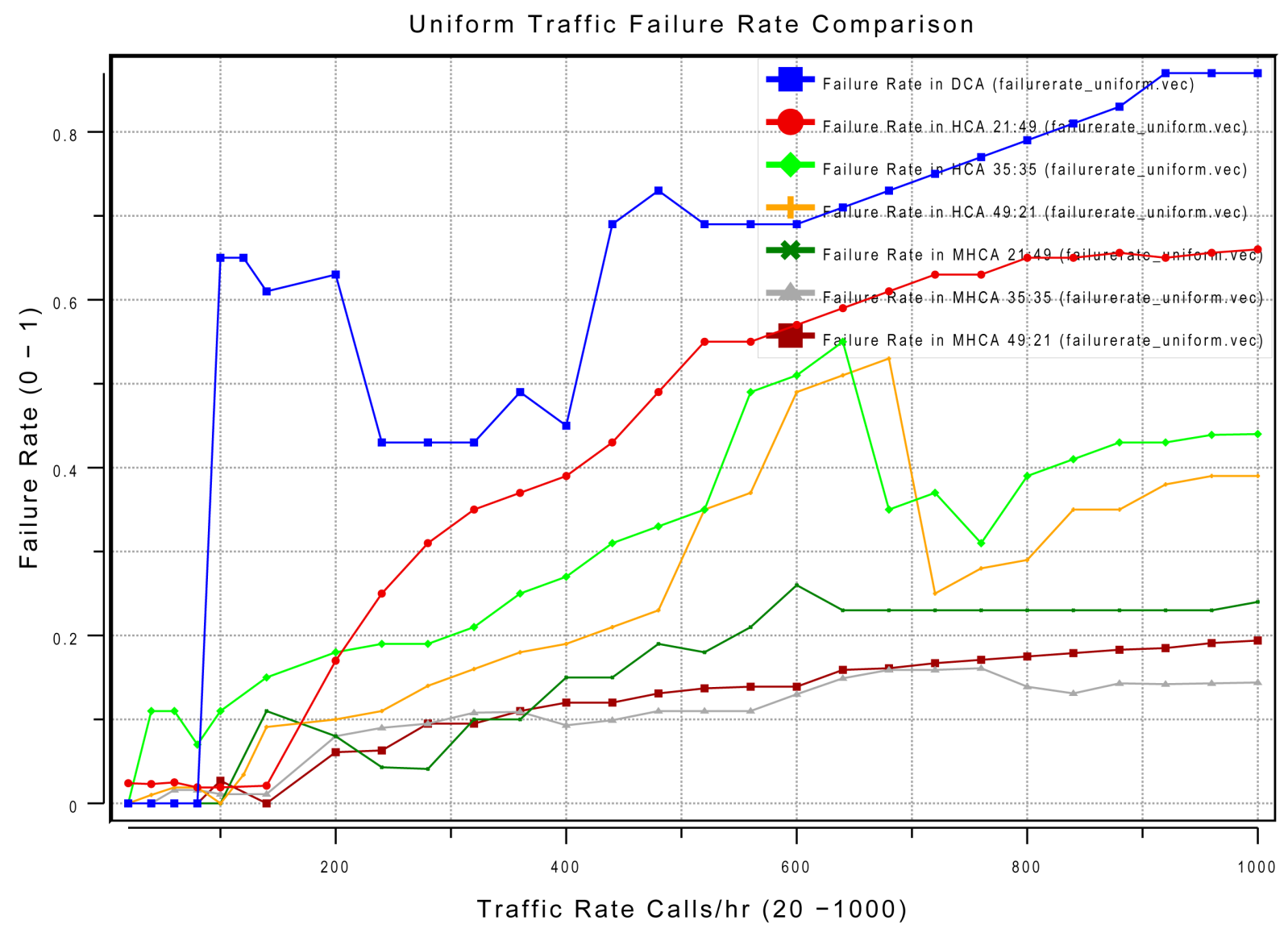

Figure 9. Failure rate comparison for uniform traffic.

Table 8. Comparison of blocking probabilities with ILP1 and ES approach [16].

\begin{tabular}{ccccccc}
\hline FC:DC & Scheme & 40 & 60 & 80 & 100 & 120 \\
\hline 2149 & ES & 0.01 & 0.02 & 0.07 & 0.14 & 0.17 \\
& ILP1 & 0.04 & 0.06 & 0.11 & 0.17 & 0.19 \\
& MA-HCA & 0.007 & 0.006 & 0.046 & 0.026 & 0.034 \\
3535 & ES & 0.01 & 0.03 & 0.08 & 0.14 & 0.17 \\
& ILP1 & 0.03 & 0.05 & 0.11 & 0.16 & 0.19 \\
& MA-HCA & 0.038 & 0.011 & 0.032 & 0.02 & 0.02 \\
& ES & 0.03 & 0.05 & 0.10 & 0.16 & 0.19 \\
& ILP1 & 0.05 & 0.07 & 0.12 & 0.18 & 0.21 \\
& MA-HCA & 0.046 & 0.0081 & 0.061 & 0.023 & 0.057 \\
\hline
\end{tabular}

ES: Evolutionary Strategy; ILP1: Integer Linear Programming 1 scheme.

cold cluster. Results have shown that proposed hybrid channel allocation using multi agent system enhances performance index of network when compared with existing hybrid channel allocation approaches.

\section{References}

[1] Papazoglou, P.M. and Karras, D.A. (2008) An Improved Multi-Agent Simulation Methodology For Modelling and Evaluating Wireless Communication Systems Resource Allocation Algorithms. Journal Of Universal Computer Science, 14, 1061-1079.

[2] Papazoglou, P.M., Karras, D.A. and Papademetriou, R.C. (2011) On Multi Agent Based Modeling and Control in 
Large Scale Wireless Communication Systems for Improved Resource Allocation Performance. Telecommunication System, 52, 1657-1675. http://dx.doi.org/10.1007/s11235-011-9658-2

[3] Lekova, A. (2012) Exploiting Mobile Ad Hoc Networking and knowledge generation to Achieve Ambient Intelligence. Applied Computational Intelligence and Soft Computing, 2012, Article ID: 262936.

[4] Elhachmi, J. and Guenoun, Z. (2011) Distributed Frequency Assignement Using Hierarchical Cooperative Multi-Agent System. International Journal of Communications, Network and System Sciences, 4, 727-734.

[5] Wu, X., Kaekel, A., Bari, A. and Ngom, A. (2010) Optimized Hybrid Resource Allocation in Wireless Cellular Networks with and without Channel Reassignment. Hindawi Publishing Corporation. Journal of Computing Systems, Networks and Communications, 2010, Article ID: 524854.

[6] Elhachimi, J. and Guennoun, Z. (2011) A Multi-Agent System for Resource Management in GSM Cellular Networks. In: Abraham, A., et al., Eds., International Symposium on DCAI, AISC 91, 99-106.

[7] Bodanese, E.L. and Cuthbert, L.G. (2000) Application of Intelligent Agents in Channel Allocation Strategies for Mobile Networks. IEEE International Conference on Communications, 1, 18-22.

[8] Iraqi, Y. and Boutaba, R. (2000) A Multi-Agent System for Resource Management in Wireless Mobile Multimedia Networks. In: Ambler, A., Calo, S.B. and Kar G., Eds., DSOM 2000, LNCS 1960, 218-229 Springer-Verlag, Berlin Heidelberg.

[9] Bigham, J. and Du, L. (2003) Cooperative Negotiation in a Multi-Agent System for Real-Time Load Balancing of a Mobile Cellular Network. AAMAS’03, Melbourne, 14-18 July 2003.

[10] Kamble, M. and Gupta, R. (2011) Multi Agent System Architecture for Admission Control and Resource Allocation in Cellular Network. Communications in Computer and Information Science, 250, 576-578. http://dx.doi.org/10.1007/978-3-642-25734-6_99

[11] Kamble, M. and Gupta, R. (2011) An Efficient Framework Using Fuzzy Logic and Multi Agent System for Cellular Network. In: Deep, K., Nagar, A., Pant, M. and Bansal, J.C., Eds., Proceedings of the International Conference on Soft Computing for Problem Solving (SocProS 2011) December 20-22, 2011, Springer, India, 513-523.

[12] Jain, P.J., et al. (2002) On Distributed Dynamic Channel Allocation in Mobile Cellular Networks. IEEE Transactions on Parallel and Distributed Systems, 13, 1024-1037.

[13] Vidyarthi, G., Ngom, A. and Stojmenovic, I. (2005) A Hybrid Channel Assignment Approach Using an Efficient Evolutionary Strategy in Wireless Network. IEEE Transactions on Vehicular Technology, 54, 1887-1895.

[14] Kamble, M. and Gupta R. (2011) A New Framework for Call Admission Control in Wireless Cellular Network. 2011 International Conference on Emerging Trends in Networks and Computer Communications (ETNCC), Udaipur, 22-24 April 2011, 178-181.

[15] Pendharkar, P.C. (2006) A Multi-Agent Distributed Channel Allocation Approach For Wireless Networks. International Transactions in Operation Research, 15, 325-337.

[16] Lee, W.C.Y. (1989) Mobile Cellular Telecommunication Systems. McGraw-Hill, New York.

[17] http://jade.tilab.com/ 
Scientific Research Publishing (SCIRP) is one of the largest Open Access journal publishers. It is currently publishing more than 200 open access, online, peer-reviewed journals covering a wide range of academic disciplines. SCIRP serves the worldwide academic communities and contributes to the progress and application of science with its publication.

Other selected journals from SCIRP are listed as below. Submit your manuscript to us via either submit@scirp.org or Online Submission Portal.
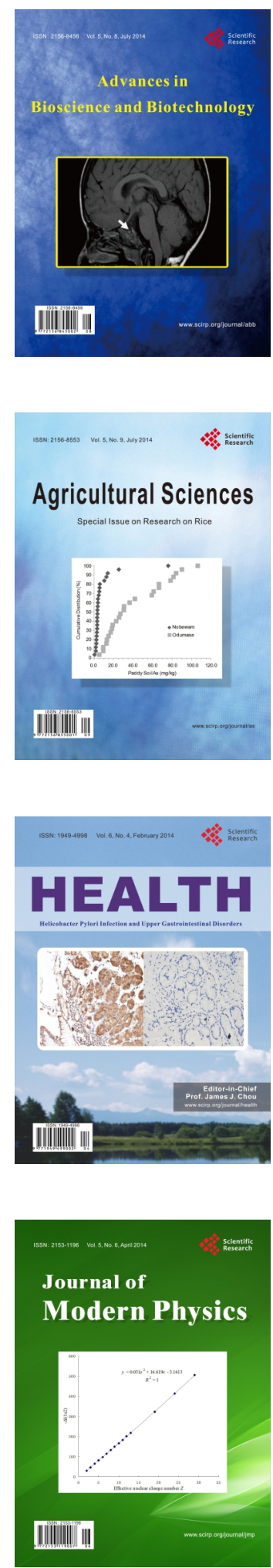
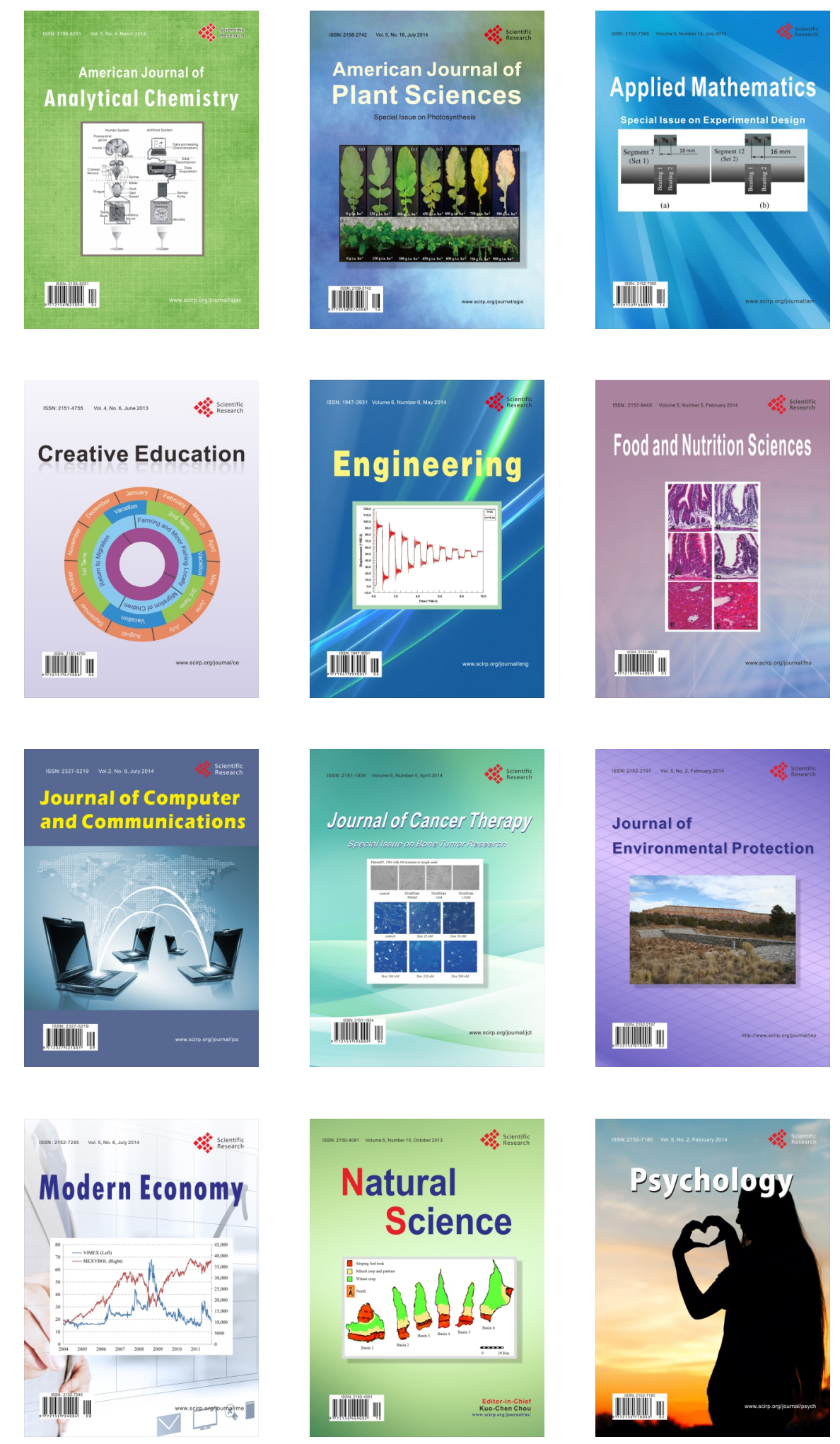